\title{
Unusual polypoid mass lesion in the rectum
}

\section{Surinder Singh Rana a , Rajender Kumar Basherb, Ravi Kumar Sharma ${ }^{a}$, Rajesh Guptac}

Postgraduate Institute of Medical Education and Research (PGIMER), Chandigarh, India

A 22-year-old male presented with constipation and bleeding per rectum. He had a history of self-digitation. Digital rectal examination revealed an irregular hard polypoid lesion in the rectum. Colonoscopy revealed multiple polypoid mass lesions in the rectum (Fig. 1A), while endoscopic biopsies were non-contributory. Positron emission computed tomography revealed a fluorodeoxyglucose-avid mass lesion in the rectum (Fig. 1B; arrow). Endoscopic ultrasound (EUS) revealed an illdefined hypoechoic lesion arising from the mucosa (Fig. 2A; arrows). The muscularis propria was not involved and mass was hard on EUS elastography (Fig. 2B; blue color; arrows). The mass was hypo-enhancing on contrast-enhanced EUS (Fig. 2C; arrows). EUS-guided fine-needle aspiration was also noncontributory. The large polypoid lesion was removed using a snare and histopathological examination revealed features suggestive of solitary rectal ulcer syndrome (SRUS). The patient was started on laxatives, behavioral therapy, and topical sucralfate. As bleeding persisted, argon plasma coagulation (APC) was used to coagulate the lesions. The bleeding subsided and sigmoidoscopy after 6 sessions of APC revealed a marked reduction in size as well as a number of polypoid lesions.

The endoscopic appearance of SRUS is variable and it may manifest as hyperemia, ulceration or a polypoid lesion that can

Department of ${ }^{\mathrm{a} G a s t r o e n t e r o l o g y ~(S u r i n d e r ~ S i n g h ~ R a n a, ~ R a v i ~ K u m a r ~}$

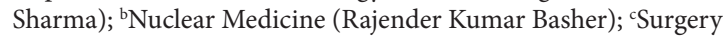
(Rajesh Gupta), Postgraduate Institute of Medical Education and Research (PGIMER), Chandigarh, India

Conflict of Interest: None

Correspondence to: Professor Surinder Singh Rana, Department of Gastroenterology, PGIMER, Chandigarh 160 012, India e-mail: drsurinderrana@yahoo.co.in

Received 10 August 2018; accepted 20 August 2018; published online 14 September 2018

DOI: https://doi.org/10.20524/aog.2018.0308
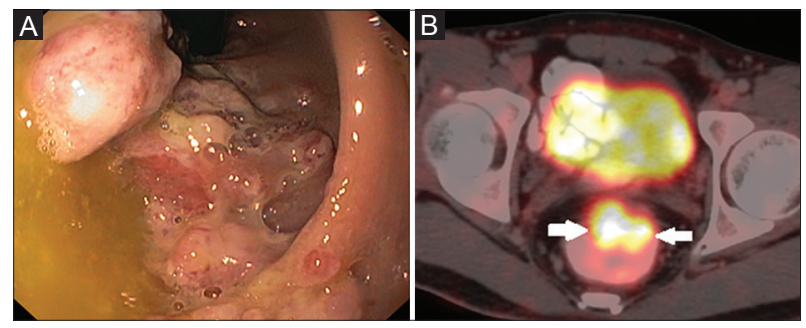

Figure 1 (A) Colonoscopy: multiple polypoid mass lesions in rectum. (B) Positron emission computed tomography: fluorodeoxyglucoseavid mass lesion in rectum (arrows)
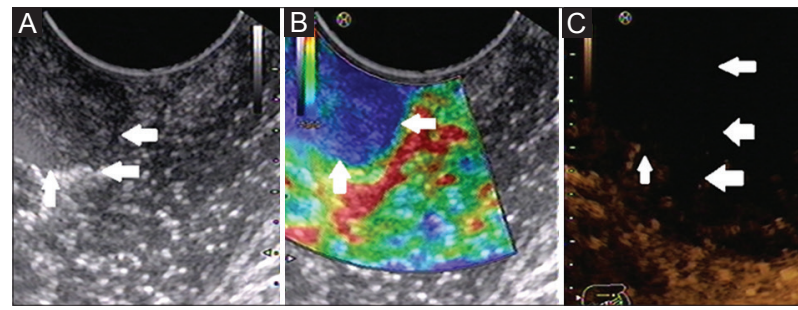

Figure 2 (A) Endoscopic ultrasound (EUS): ill-defined hypoechoic lesion arising from the mucosa (arrows). (B) Mass was hard on EUS elastography (blue color; arrows). (C) Mass was hypo-enhancing on contrast-enhanced EUS (arrows)

mimic cancer [1]. EUS findings of polypoid SRUS have not been described but, being fibrotic, it should be hard and hypoenhancing, as in the index case.

\section{Reference}

1. Saadah OI, Al-Hubayshi MS, Ghanem AT. Solitary rectal ulcer syndrome presenting as polypoid mass lesions in a young girl. World J Gastrointest Oncol 2010;2:332-334. 